TIFR/TH/97-20

\title{
New Source Term for QGP Formation in the Background-Field Model
}

\author{
R. S. Bhalerao \\ Theoretical Physics Group \\ Tata Institute of Fundamental Research \\ Homi Bhabha Road, Colaba, Mumbai 400 005, India \\ V. Ravishankar \\ Department of Physics \\ Indian Institute of Technology \\ Kanpur 208 016, India
}

\begin{abstract}
We consider pair production in a space-time-dependent background field and derive a source term, i.e., production rate in the one-particle phase space. Such a source term is required in Boltzmann-equation-based models of quarkgluon plasma formation and evolution. We compare the source term derived here with the one that has been used in the literature so far. Significant differences are observed.
\end{abstract}

PACS: 12.38.Mh, 25.75.-q, 25.75.Dw

Keywords: relativistic heavy-ion collisions, quark-gluon plasma, pair production from spacetime-dependent background field, preequilibrium phenomena

E-mail: bhalerao@theory.tifr.res.in, $\quad$ Fax:09122 2152110

E-mail: vravi@iitk.ernet.in, $\quad$ Fax:0910512 250260 


\section{INTRODUCTION}

In some models of ultrarelativistic nucleus-nucleus collisions, the formation of quarkgluon plasma (QGP) is assumed to proceed via creation of a strong colour-electric field in the region between the two nuclei receding from each other after the collision, and the subsequent decay of the field by parton-pair production according to the well known nonperturbative formula given by Schwinger [1]. We shall call these models [2 7] background-field models. They make use of the framework of the relativistic transport theory to study the formation and preequilibrium evolution of QGP, with Schwinger's formula providing a basis for derivation of the source term (i.e., production rate in the one-particle phase space) in the Boltzmann transport equation.

In analogy with an ordinary parallel-plates capacitor, the above models have also been called colour-plates models. The colour field, however, need not owe its existence entirely to the colour plates. A high-density many-body system is often conveniently described in terms of a self-consistent mean field and residual short-range interactions. For example, Blaizot and Iancu [8] have considered gauge theories at high temperatures and shown that long-wavelength excitations of QGP can be described as collective oscillations of gauge and fermionic mean fields. They have presented non-abelian solutions to the equations of motion, which correspond to spatially uniform colour oscillations. Importance of the mean field has also been stressed in the context of hot and dense hadron gas models of relativistic heavy ion collisions; see for example [9]. Whatever may be the origin of the colour field, once it is there, it can produce pairs of partons, add to the entropy of the system and affect its subsequent evolution.

A few remarks on the source term mentioned above are in order. The exact one-loop nonperturbative result (Fig. 1a) that Schwinger derived for pair production in a constant, uniform background electric field $(E)$ is given by

$$
\frac{d W}{d^{3} x d t}=\frac{\alpha E^{2}}{\pi^{2}} \sum_{n=1}^{\infty} \frac{1}{n^{2}} \exp \left(-\frac{n \pi m^{2}}{|e E|}\right),
$$


where $\alpha=e^{2} / 4 \pi$ is the fine-structure constant and $m$ is the particle mass. It is instructive to understand this result in terms of a semiclassical tunneling across the mass gap [10]. This result, strictly speaking, is applicable to the parton pair production only in the so-called abelian approximation. Secondly, Eq. (1.1) gives the probability per unit volume and per unit time, that a pair is produced, while the transport equation requires the source term in one-particle phase space. Finally, and more importantly, unlike the case considered in [1] where the constant electric field is maintained by external sources, the pairs produced in the background-field models derive their energy and momentum from the field, the field becomes time dependent and Eq. (1.1) stops being applicable.

The resolution of these problems has so far involved rewriting the right-hand side of Eq. (1.1) as an integral over $p_{T}^{2}$ or $m_{T}^{2} \equiv p_{T}^{2}+m^{2}, p_{T}$ being the parton momentum transverse to the direction of the collision axis. The expression so obtained has often been used in the QGP transport equation and it reads 10

$$
\frac{d W}{d^{3} x d t d^{2} p_{T}}=\frac{1}{4 \pi^{3}}\left|e E \ln \left[1-\exp \left(-\frac{\pi m_{T}^{2}}{|e E|}\right)\right]\right| .
$$

While the above extension may be justified semiclassically, the next assumption that the constant $E$ in Eq. (1.2), may be replaced by its time-dependent counterpart $E(t)$, merits a critical examination. Strictly speaking, this replacement is invalid since as soon as the field acquires time dependence, it can directly excite negative-energy particles to levels above the mass gap, by a perturbative mechanism, without recourse to any tunneling or barrier penetration mechanism. The above replacement may be justified as a kind of a localfield approximation provided the time-dependence of $E(t)$ is so weak that it only excites frequencies with magnitudes negligible compared to the mass gap. Equivalently, the field needs to be constant on the scale of the Compton wavelength $m^{-1}$. Fields that are constant over such large time intervals are unlikely to be produced in heavy-ion collisions. Moreover, a justification for the local-field approximation, which could be looked for a posteriori is not forthcoming from the explicit numerical results presented in [5].

The purpose of this paper is to take the time dependence of the electric field into account, 
as properly as a semiclassical formalism would allow, and present a new source term based on Fig. 1b, for use in the QGP transport equation, thereby avoiding the unphysical aspect that we have pointed out in the current formulation. The mechanism depicted in Fig. 1b was used by Martin et al. [11] to calculate the total energy deposited during a certain time interval, under certain assumptions. They did not derive a source term.

\section{NEW SOURCE TERM}

For fermion pair production in the presence of a space-time-dependent background field the amplitude to the lowest order (Fig. 1b) is given by

$$
\text { -ie } \bar{u}\left(p_{1}\right) A(K) v\left(p_{2}\right)
$$

and the probability for pair production is

$$
W=\frac{\alpha}{\pi} \int d^{4} K \delta^{(4)}\left(K-p_{1}-p_{2}\right) \int \frac{d^{3} p_{1}}{2 E_{1}} \frac{d^{3} p_{2}}{2 E_{2}} T
$$

where

$$
T \equiv \operatorname{tr}\left[(\not p 1+m) A(K)\left(\not p_{2}-m\right) \not A(-K)\right]
$$

Since our aim is to obtain the production rate in the one-particle phase space, for insertion in the Boltzmann equation for a one-particle distribution function $f(x, p)$, we choose to perform the $K$-integration first. Next, in order to evaluate $T$, we introduce

$$
P \equiv p_{1}+p_{2} \text { and } p \equiv p_{1}-p_{2}
$$

and use $p_{1} \cdot p_{2}+m^{2}=P^{2} / 2$. This gives

$$
\begin{aligned}
T & =(P+p) \cdot A(P)(P-p) \cdot A(-P)-2 P^{2} A(P) \cdot A(-P)+(P+p) \cdot A(-P)(P-p) \cdot A(P) \\
& =2 P \cdot A(P) P \cdot A(-P)-2 P^{2} A(P) \cdot A(-P)-2 p \cdot A(P) p \cdot A(-P)
\end{aligned}
$$

Now recall 


$$
\begin{aligned}
-\frac{1}{2} F_{\mu \nu}(P) F^{\mu \nu}(-P) & =P \cdot A(P) P \cdot A(-P)-P^{2} A(P) \cdot A(-P) \\
& =|\mathbf{E}(P)|^{2}-|\mathbf{B}(P)|^{2} .
\end{aligned}
$$

To be able to compare our results with those based on Eq. (1.2), we ignore the magnetic field $\mathbf{B}(P)$. Note also that pair production is an electric effect [1]. Equations (2.3) and (2.4) give us

$$
T=2|\mathbf{E}(P)|^{2}-2 p \cdot A(P) p \cdot A(-P) .
$$

Note that the remaining term $p \cdot A(P) p \cdot A(-P)$ in Eq. (2.5) is also invariant under the gauge transformation

$$
A_{\mu}(P) \rightarrow A_{\mu}(P)-i P_{\mu} \chi(P)
$$

because $p \cdot P=0$. We shall now rewrite this term also in terms of $\mathbf{E}(P)$. To that end, we choose the following gauge:

$$
\begin{aligned}
A^{1} & =0=A^{2}, \\
A^{\alpha}(x) & =\epsilon^{\alpha \beta} \partial_{\beta} a(x), \quad(\alpha, \beta=0 \text { or } 3)
\end{aligned}
$$

where $a(x)=a(t, z)$ is an arbitrary real function vanishing at $t$ and $z= \pm \infty$, and $\epsilon^{\alpha \beta}$ is the totally antisymmetric Levi-Civita tensor in $(1+1)$ dimensions. (This gauge choice is natural in the colour-plates model where $\mathbf{E}$ is taken along the collision or $z$-axis.) Equation (2.6) ensures the gauge condition $\partial_{\mu} A^{\mu}(x)=0$. We have

$$
\begin{aligned}
A^{\alpha}(P) & =(2 \pi)^{-2} \int d^{4} x \exp (-i P \cdot x) A^{\alpha}(x) \\
& =i \epsilon^{\alpha \beta} P_{\beta}(2 \pi)^{-2} \int d^{4} x \exp (-i P \cdot x) a(x) \\
& =i \epsilon^{\alpha \beta} P_{\beta} a(P) \\
& \equiv i \tilde{P}^{\alpha} a(P),
\end{aligned}
$$

where $\tilde{P}$ is the dual of $P$. 
Keeping in mind the boost-invariant central-rapidity region expected to be formed in ultrarelativistic heavy-ion collisions, we assume that the electric field $\mathbf{E}(x)$ depends only on the boost-invariant variable $\tau \equiv \sqrt{t^{2}-z^{2}}$. Hence

$$
\begin{aligned}
\mathbf{E}(P) & =(2 \pi)^{-2} \int d^{4} x \exp (-i P \cdot x) \mathbf{E}(x) \\
& =\delta^{(2)}\left(\mathbf{P}_{T}\right) \int d t d z \exp \left[-i\left(P_{0} t-P_{z} z\right)\right] \mathbf{E}(\tau)
\end{aligned}
$$

It follows that $\mathbf{p}_{1 T}=-\mathbf{p}_{2 T}$ and hence the two particles have identical transverse mass, say $m_{T}$. The relevant components of their 4-momenta are

$$
p_{1}^{\alpha}=m_{T}\left(\cosh y_{1}, \sinh y_{1}\right), \quad p_{2}^{\alpha}=m_{T}\left(\cosh y_{2}, \quad \sinh y_{2}\right)
$$

such that

$$
\tilde{p}_{1}^{\alpha}=-m_{T}\left(\sinh y_{1}, \cosh y_{1}\right), \quad \tilde{p}_{2}^{\alpha}=-m_{T}\left(\sinh y_{2}, \cosh y_{2}\right),
$$

where $y_{1,2}$ are their rapidities. For a future reference we note the following results

$$
\begin{aligned}
p_{1} \cdot \tilde{p}_{1} & =0=p_{2} \cdot \tilde{p}_{2}, \quad \tilde{p}_{1} \cdot p_{2}=-p_{1} \cdot \tilde{p}_{2}, \\
P \cdot \tilde{P} & =0, \quad \tilde{P}^{2}=-P^{2}, \\
P^{2} & =\left(p_{1}+p_{2}\right)^{2}=2 m_{T}^{2}\left[1+\cosh \left(y_{1}-y_{2}\right)\right] .
\end{aligned}
$$

Now in order to rewrite $p \cdot A(P) p \cdot A(-P)$ in terms of $\mathbf{E}(P)$, we use Eqs. (2.7), (2.2), (2.10) and (2.9) to get

$$
p \cdot A(P) p \cdot A(-P)=4 m_{T}^{4} \sinh ^{2}\left(y_{1}-y_{2}\right) a(P) a(-P) .
$$

We also note from Eqs. (2.4), (2.7) and (2.11) that

$$
|\mathbf{E}(P)|^{2}=\left(P^{2}\right)^{2} a(P) a(-P)
$$

Finally, eliminating $a(P) a(-P)$ between Eqs. (2.13) and (2.14) we get the desired expression

$$
p \cdot A(P) p \cdot A(-P)=4 m_{T}^{4} \sinh ^{2}\left(y_{1}-y_{2}\right)|\mathbf{E}(P)|^{2} /\left(P^{2}\right)^{2} .
$$


The occurrence of $\left(P^{2}\right)^{2}$ in the denominator is noteworthy. It indicates a highly nonlocal nature of this term in the configuration space; the production rate depends not only on the instantaneous strength of the field but also on its derivatives. This feature is absent in Eq. (1.2). Substituting $P^{2}$ from Eq. (2.12) in Eq. (2.15) and then substituting the resulting expression in Eq. (2.5) we get

$$
T=2|\mathbf{E}(P)|^{2} \operatorname{sech}^{2}\left(\frac{y_{1}-y_{2}}{2}\right)
$$

Thus the probability $W$ in Eq. (2.1) becomes

$$
W=\frac{\alpha}{2 \pi} \int \frac{d^{3} p_{1}}{E_{1}} \frac{d^{3} p_{2}}{E_{2}}|\mathbf{E}(P)|^{2} \operatorname{sech}^{2}\left(\frac{y_{1}-y_{2}}{2}\right) .
$$

In order to evaluate $\mathbf{E}(P)$ occurring in Eq. (2.16) we define (for $t>0, t^{2}>z^{2}$ )

$$
\begin{aligned}
\tau^{2} & =t^{2}-z^{2}, \quad \eta=\tanh ^{-1}(z / t), \\
M^{2} & =P_{0}^{2}-P_{z}^{2}, \quad Y=\tanh ^{-1}\left(P_{z} / P_{0}\right),
\end{aligned}
$$

and substitute

$$
\begin{aligned}
d t d z & =\tau d \tau d \eta \\
P_{0} t-P_{z} z & =M \tau \cosh (Y-\eta) \equiv M \tau \cosh \theta
\end{aligned}
$$

in Eq. (2.8) to get

$$
E(P)=\delta^{(2)}\left(\mathbf{P}_{T}\right) \int_{-\infty}^{\infty} d \theta \int_{0}^{\infty} d \tau \tau \exp (-i M \tau \cosh \theta) E(\tau)
$$

We shall assume for the sake of definiteness and simplicity that $E(\tau)$ depends on only one time scale $\left(\tau_{0}\right)$ and is of the form

$$
E(\tau)=E_{0} \exp \left(-\tau / \tau_{0}\right), \quad \tau_{0}>0
$$

Self-consistent numerical calculations reported in Ref. [5] predict $\tau$-dependence which is consistent with Eq. (2.18). Substituting $E(\tau)$ given in Eq. (2.18) in Eq. (2.17) and performing the $\tau$-integration by parts, we get 


$$
E(P)=E_{0} \delta^{(2)}\left(\mathbf{P}_{T}\right) \tau_{0}^{2} \int_{-\infty}^{\infty} d \theta\left(1+i M \tau_{0} \cosh \theta\right)^{-2}
$$

The $\theta$-integration too can be done analytically [12] which gives

$$
E(P)=E_{0} \delta^{(2)}\left(\mathbf{P}_{T}\right) \tau_{0}^{2} 2 I\left(M \tau_{0}\right) /\left(1+M^{2} \tau_{0}^{2}\right)
$$

where

$$
I(x) \equiv-1+\frac{1}{\sqrt{1+x^{2}}} \ln \frac{1+i x+\sqrt{1+x^{2}}}{1+i x-\sqrt{1+x^{2}}} .
$$

We continue with our derivation of $W$ by substituting $E(P)$ from Eq. (2.19) in Eq. (2.16). This entails the calculation of $\left[\delta^{(2)}\left(\mathbf{P}_{T}\right)\right]^{2}$ which we perform as follows:

$$
\begin{aligned}
{\left[\delta^{(2)}\left(\mathbf{P}_{T}\right)\right]^{2} } & =\delta^{(2)}\left(\mathbf{P}_{T}\right) \int \exp \left(i \mathbf{P}_{T} \cdot \mathbf{x}_{T}\right) d^{2} x_{T} /(2 \pi)^{2} \\
& \rightarrow \delta^{(2)}\left(\mathbf{P}_{T}\right) d^{2} x_{T} /(2 \pi)^{2}
\end{aligned}
$$

This is a standard procedure and it allows us to write the probability per unit transverse area. Next, recall the occurrence of $\left(P^{2}\right)^{2}$ in Eq. (2.15) and the comment made thereafter. In order to recover the dependence of $W$ on the instantaneous strength of the field $|E(\tau)|^{2}$ and to obtain a time-dependent rate, we shall employ the integral representation:

$$
\frac{1}{2} \tau_{0}=\int_{0}^{\infty} \exp \left(-2 \tau / \tau_{0}\right) d \tau
$$

Equations (2.19)-(2.22) together with Eq. (2.16) yield

$$
\begin{aligned}
d W=\alpha\left(\frac{\tau_{0}}{\pi}\right)^{3} & d^{2} x_{T} d \tau|E(\tau)|^{2} \int \frac{d^{3} p_{1}}{E_{1}} \frac{d^{3} p_{2}}{E_{2}} \delta^{(2)}\left(\mathbf{P}_{T}\right) \\
& \times\left|I\left(M \tau_{0}\right)\right|^{2} \operatorname{sech}^{2}\left(\frac{y_{1}-y_{2}}{2}\right)\left(1+M^{2} \tau_{0}^{2}\right)^{-2}
\end{aligned}
$$

Note that

$$
\frac{d^{3} p_{1}}{E_{1}} \frac{d^{3} p_{2}}{E_{2}}=d^{2} p_{1 T} d y_{1} d^{2} p_{2 T} d y_{2}
$$

The integration over $\mathbf{p}_{2 T}$ can be done trivially with the help of the delta function $\delta^{(2)}\left(\mathbf{P}_{T}=\right.$ $\left.\mathbf{p}_{1 T}+\mathbf{p}_{2 T}\right)$. In order to obtain the production rate in the one-particle phase space we follow 
the literature and assume as in Ref. [2] that if a particle is produced at $z$ and $t$, it must appear with the longitudinal velocity $p_{z} / E=z / t$. (This is reminiscent of the ThomasFermi approximation in condensed-matter physics.) It allows us to identify the spatial and momentum rapidities and we can write $d \tau d y_{1}=d t d z / \tau$.

Combining these results we finally get the new source term:

$$
\frac{d W}{d^{3} x d t d^{2} p_{T}}=\alpha\left(\frac{\tau_{0}}{\pi}\right)^{3} \frac{|E(\tau)|^{2}}{\tau} \int_{-\infty}^{\infty}\left|I\left(M \tau_{0}\right)\right|^{2} \operatorname{sech}^{2}\left(\frac{y_{1}-y_{2}}{2}\right)\left(1+M^{2} \tau_{0}^{2}\right)^{-2} d\left(y_{1}-y_{2}\right),
$$

where the electric field $E(\tau)$ and the function $I\left(M \tau_{0}\right)$ are defined in Eqs. (2.18) and (2.20), respectively, and from Eq. (2.12) the invariant mass of the pair, $M$, is given by

$$
M=2 m_{T} \cosh \frac{1}{2}\left(y_{1}-y_{2}\right) .
$$

\section{RESULTS}

We now compare the old and the new source terms given in Eqs. (1.2) and (2.23), respectively. Both are invariant under Lorentz boosts in the longitudinal or $z$ direction. In the limit $E \rightarrow 0,(1.2)$ and all of its derivatives vanish, while (2.23) behaves like $\sim E^{2}$. In the large $E$ limit, (1.2) behaves like $\sim E \ln (E)$, while (2.23) behaves like $\sim E^{2}$. Thus the ratio of the new and the old source terms increases with the strength of the electric field. The transverse-momentum $\left(p_{T}\right)$ dependence arises naturally in the derivation of Eq. (2.23). The longitudinal-momentum dependence too can be recovered, if necessary.

We also studied these two terms numerically as a function of the proper time $\tau$ and the transverse momentum $p_{T}$, for various values of the input parameters, namely the particle mass $m$, the parameter $\tau_{0}$ appearing in Eq. (2.18), the fine-structure constant $\alpha$, and the initial strength of the electric field $E_{0}$. Some representative results are shown in Figs. (2)(4), where we have taken $m=5 \mathrm{MeV}, \tau_{0}=0.1 \mathrm{fm}, \alpha=0.2$, and $\frac{1}{2} E_{0}^{2}=10 \mathrm{GeV} / \mathrm{fm}^{3}$. Significant differences are evident. 
In view of the above comments and the numerical results presented here, the two source terms, Eqs. (1.2) and (2.23), when substituted in the Boltzmann equation are expected to contribute differently to the formation of the quark-gluon plasma. It is straightforward to extend Eq. (2.23) to the case of coloured quark and antiquark production, by introducing an appropriate colour factor. A similar source term for gluon production in a space-timedependent colour field would also be required; that work is in progress 13 . It would be interesting to substitute these new source terms in a nonabelian transport equation [14], and study formation of quark-gluon plasma. We plan to undertake this work in the future.

\section{ACKNOWLEDGMENTS}

We thank M. Guchait and N. Parua for drawing the diagrams in Fig. 1. 


\section{REFERENCES}

[1] J. Schwinger, Phys. Rev. 82, 664 (1951).

[2] K. Kajantie and T. Matsui, Phys. Lett. B 164, 373 (1985).

[3] G. Gatoff, A. K. Kerman and T. Matsui, Phys. Rev. D 36, 114 (1987).

[4] A. Bialas, W. Czyz, A. Dyrek and W. Florkowski, Nucl. Phys. B 296, 611 (1988).

[5] B. Banerjee, R. S. Bhalerao and V. Ravishankar, Phys. Lett. B 224, 16 (1989).

[6] M. Asakawa and T. Matsui, Phys. Rev. D 43, 2871 (1991).

[7] J. M. Eisenberg, e-print hep-ph/9609205 and references therein.

[8] J.-P. Blaizot and E. Iancu, Nucl. Phys. B 417, 608 (1994); Phys. Rev. Lett. 72, 3317 (1994).

[9] J. M. Haeuser, W. Cassing and A. Peter, Nucl. Phys. A 585, 727 (1995) and references therein; B.-A. Li and C. M. Ko, Phys. Rev. C 52, 2037 (1995).

[10] A. Casher, H. Neuberger and S. Nussinov, Phys. Rev. D 20, 179 (1979).

[11] C. Martin, D. Vautherin and J. Cugnon, Phys. Lett. B 227, 301 (1989).

[12] I. S. Gradshteyn and I. M. Ryzhik, Table of Integrals, Series, and Products, 5th Edition, (Academic Press, San Diego, 1994), p. 131.

[13] G. C. Nayak and V. Ravishankar, in preparation.

[14] G. C. Nayak and V. Ravishankar, Phys. Rev. D, in press. 


\section{FIGURES}

FIG. 1. Schematic diagrams for pair production in (a) time-independent and (b) time-dependent electric fields. In (a), crosses indicate interactions (to all orders) with the vacuum and the vertical line represents the "cut". (b) represents pair production in the lowest-order perturbation theory.

FIG. 2. The old source term, Eq. (1.2), in units of $\mathrm{fm}^{-4} \mathrm{GeV}^{-2}$, as a function of the transverse momentum $p_{T}(\mathrm{GeV})$, for various values of the proper time $\tau$. The curves are labelled by $\tau(\mathrm{fm})$. Input parameters are as in the text.

FIG. 3. Same as Fig. 2, for the new source term, Eq. (2.23). Note the difference in the scale.

FIG. 4. The ratio of the new and the old source terms. The curves are labelled by $\tau(\mathrm{fm})$. 


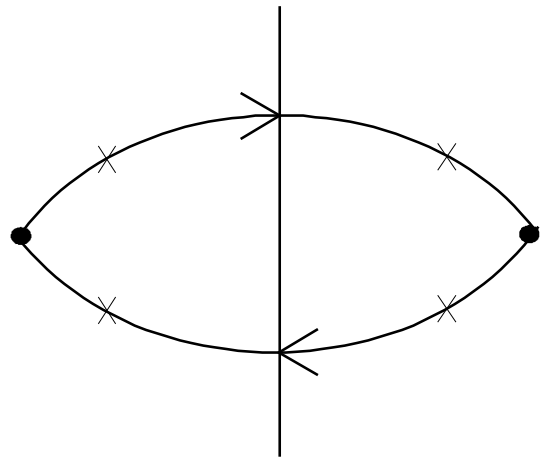

(a)

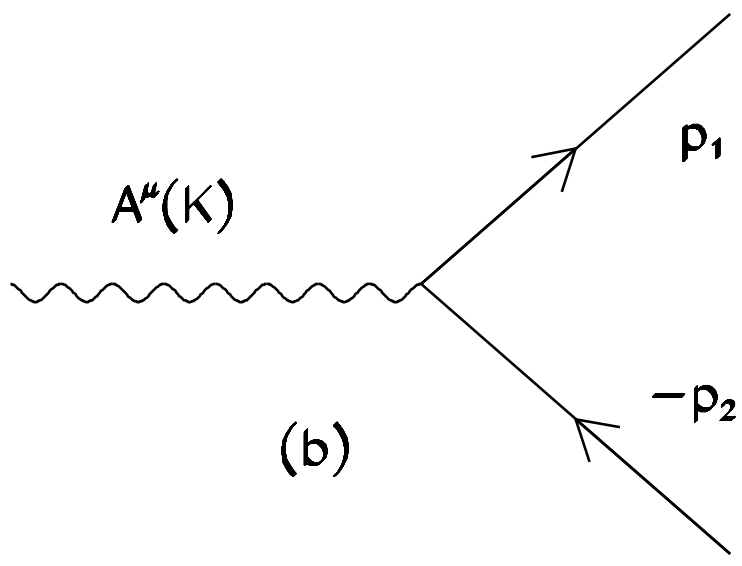

Fig. 1 
Fig. 2

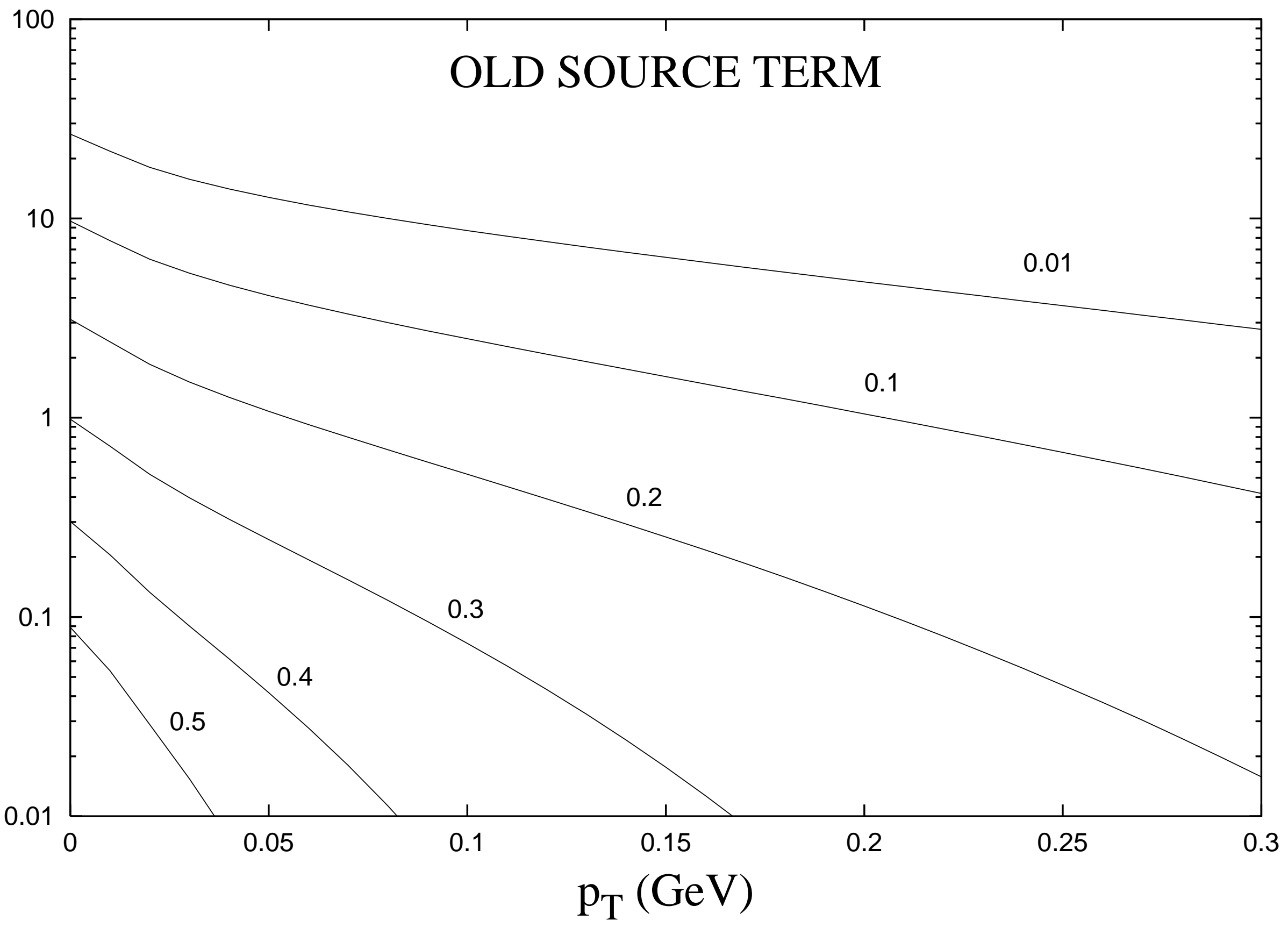


Fig. 3

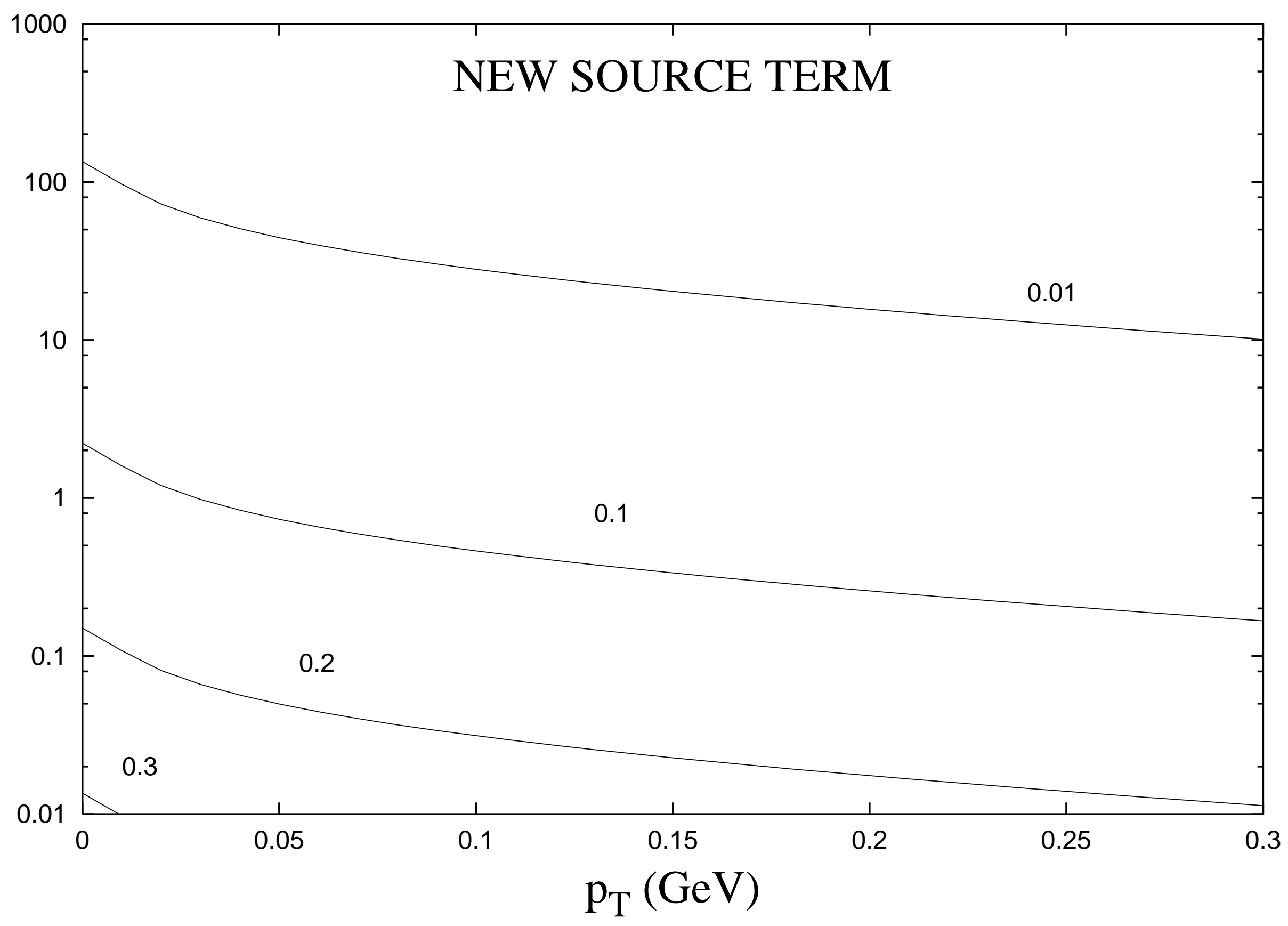


Fig. 4

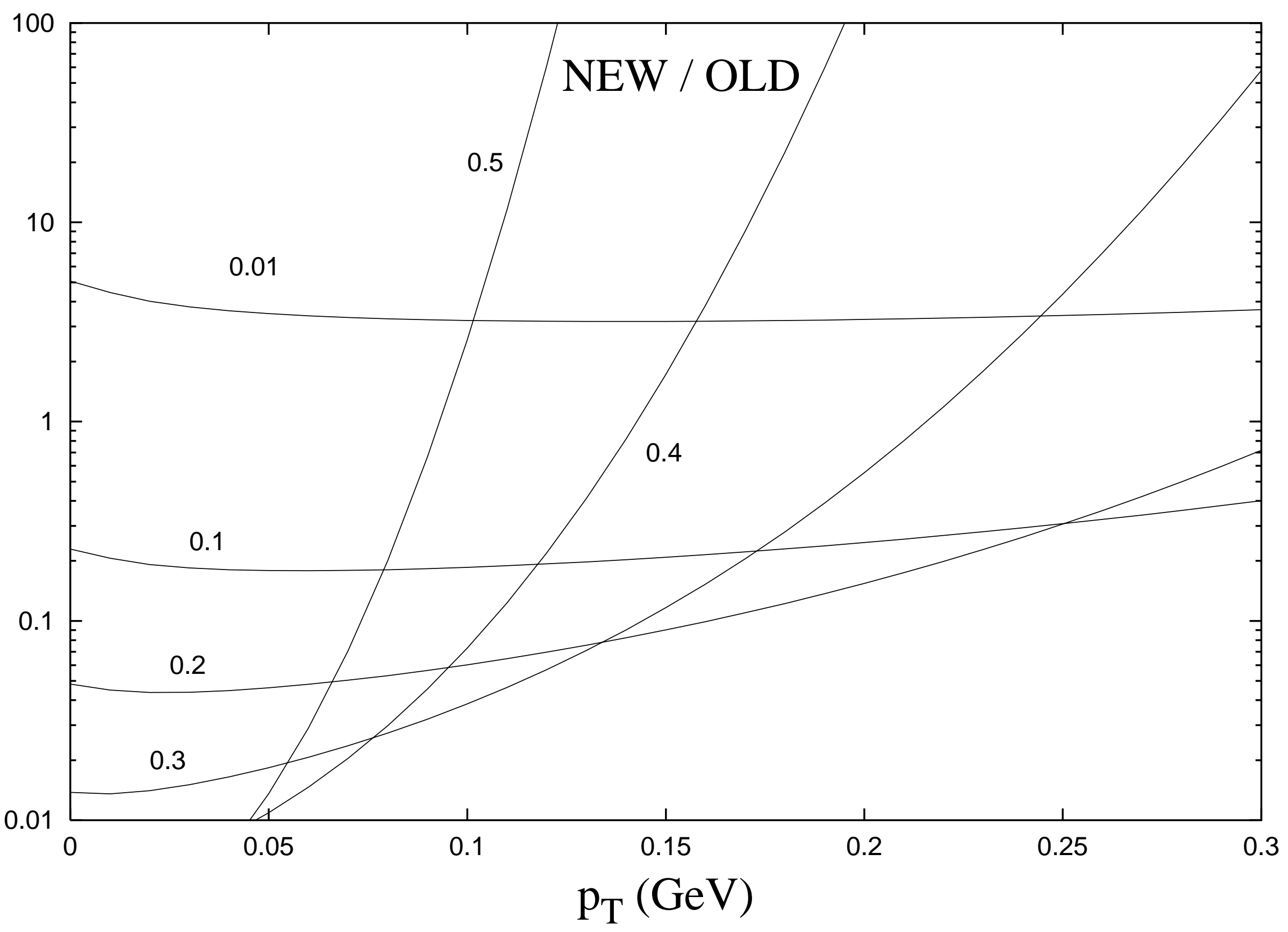

